\title{
Interquark potential with finite quark mass from lattice QCD
}

\author{
Taichi Kawana: and Shoichi Sasak \\ Department of Physics, The University of Tokyo, \\ Hongo 7-3-1, Tokyo 113-0033, Japan
}

(Dated: October 25, 2018)

\begin{abstract}
We present an investigation of the interquark potential determined from the $q \bar{q}$ Bethe-Salpeter (BS) amplitude for heavy quarkonia in lattice QCD. The $q \bar{q}$ potential at finite quark mass $m_{q}$ can be calculated from the equal-time and Coulomb gauge BS amplitude through the effective Schrödinger equation. The definition of the potential itself requires information about a kinetic mass of the quark. We then propose a self-consistent determination of the quark kinetic mass on the same footing. To verify the proposed method, we perform quenched lattice QCD simulations with a relativistic heavy-quark action at a lattice cutoff of $1 / a \approx 2.1 \mathrm{GeV}$ in a range of the quark kinetic mass, $1.0 \leq m_{q} \leq 3.6 \mathrm{GeV}$. Our numerical results show that the $q \bar{q}$ potential in the infinitely heavyquark limit $\left(m_{q} \rightarrow \infty\right)$ is fairly consistent with the conventional one obtained from Wilson loops. The quark-mass dependence of the $q \bar{q}$ potential and the spin-spin potential are also examined.
\end{abstract}

One of the major successes of lattice QCD is to demonstrate that a confining potential naturally emerges between a static, infinitely heavy quark $(q)$ and antiquark $(\bar{q})$ by considering the Wilson loop [1]. This static $q \bar{q}$ potential can be well parametrized by the Coulomb plus linear potential, which resembles the phenomenology of confining quark interactions, the so-called Cornell potential [2], adopted for studying heavy-quark bound systems such as charmonia and bottomonia [1, 3, 4. However, there is still a gap between the static $q \bar{q}$ potential expressed in terms of Wilson loops and the phenomenological heavy-quark potential in quark models. The former is calculated for infinitely heavy quarks, while the latter is exploited at heavy, but finite quark mass.

Recently, the leading and next-to-leading order corrections, which are classified in powers of the inverse of heavy-quark mass $m_{q}$ or powers of the relative quark velocity $v$ within a framework called potential nonrelativistic QCD 5, to the static $q \bar{q}$ potential given by Wilson loops have been successfully calculated in quenched lattice QCD with high accuracy by using a multilevel algorithm [6, 7]. Although the $1 / m_{q}$ (or $v$ ) expansion in the Wilson loop formalism could be continued systematically to higher orders, where the bottom quark can be treated, this methodology is no longer applicable at the mass of the charm quark, where the motion of the quark and antiquark should not be small enough to be perturbatively treated [1].

How can we incorporate the effect stemming from the motion of the quark and antiquark beyond the perturbative treatment? Ikeda and Iida have recently proposed an interesting idea that the $q \bar{q}$ interquark potential at finite quark mass can be calculated within the Bethe-Salpeter (BS) amplitude approach [8, which is originally applied for the hadron-hadron interactions including the first attempt of the nuclear force 9]. However, the definition of the interquark potential itself requires extra information of the quark mass.

In this letter, we propose the self-consistent determina- tion of the quark kinetic mass within this BS amplitude approach. The quark kinetic mass $m_{q}$ is defined through the large-distance behavior in the spin-dependent part of the interquark potential with the help of the measured hyperfine splitting of $1 S$ states in heavy quarkonia. Then, we can define a proper interquark potential at finite quark mass, which potentially accounts for all orders of $1 / m_{q}$ corrections.

Let us briefly review the new method utilized here to calculate the interquark potential $V_{q \bar{q}}$ at finite quark mass. A gauge-invariant definition of the equal-time $q \bar{q}$ BS amplitude for quarkonium states is given by

$$
\phi_{\Gamma}(\mathbf{r})=\sum_{\mathbf{x}}\left\langle 0|\bar{q}(\mathbf{x}) \Gamma \mathcal{M}(\mathbf{x}, \mathbf{x}+\mathbf{r}) q(\mathbf{x}+\mathbf{r})| q \bar{q} ; J^{P}\right\rangle
$$

where $\mathbf{r}$ is the relative coordinate of two quarks at a certain time slice, $\Gamma$ is chosen as $\gamma_{5}$ for the pseudoscalar (PS) state $\left(J^{P}=0^{-}\right)$, and $\gamma_{i}$ for the vector $(\mathrm{V})$ state $\left(J^{P}=1^{-}\right)$[11, 12. $\mathcal{M}$ is a path-ordered product of gauge links. In the Coulomb or Landau gauge, the BS amplitude can be simply evaluated with $\mathcal{M}=1$. Hereafter, we consider the Coulomb gauge BS amplitude. This amplitude is given by the following four-point correlation function:

$$
\begin{aligned}
& G_{\Gamma}\left(\mathbf{r}, t ; t_{\mathrm{src}}\right) \\
= & \sum_{\mathbf{x}, \mathbf{x}^{\prime}, \mathbf{y}^{\prime}}\left\langle\bar{q}(\mathbf{x}, t) \Gamma q(\mathbf{x}+\mathbf{r}, t)\left(\bar{q}\left(\mathbf{x}^{\prime}, t_{\mathrm{src}}\right) \Gamma q\left(\mathbf{y}^{\prime}, t_{\mathrm{src}}\right)\right)^{\dagger}\right\rangle
\end{aligned}
$$

where both quark and antiquark at source location $\left(t_{\mathrm{src}}\right)$ are separately projected onto a zero-momentum state by a summation over all spatial coordinates $\mathbf{x}^{\prime}$ and $\mathbf{y}^{\prime}$. Suppose that $\left|t-t_{\mathrm{src}}\right| / a \gg 1$ is satisfied, the four-point correlation function asymptotically behaves as

$$
G_{\Gamma}\left(\mathbf{r}, t ; t_{\mathrm{src}}\right) \propto \phi_{\Gamma}(\mathbf{r}) e^{-M_{\Gamma}\left(t-t_{\mathrm{src}}\right)},
$$

where $M_{\Gamma}$ is the rest mass for the ground state of heavy quarkonia and the $\mathbf{r}$-dependent amplitude corresponds 
to the Coulomb gauge BS amplitude, namely, the BS wave function [11, 12. After an appropriate projection with respect to discrete rotation, we can get the BS wave function projected in the $A_{1}^{+}$representation, $\phi_{\Gamma}(\mathbf{r}) \rightarrow \phi_{\Gamma}\left(A_{1}^{+} ; r\right)$, which corresponds to the $S$ wave in continuum theory at low energy. Details of the $A_{1}^{+}$projection are described in Ref. [13. We simply denote the $A_{1}^{+}$projected BS wave function by $\phi_{\Gamma}(r)$ hereafter.

The interquark potential $V_{\Gamma}$ can be determined from the projected BS wave function $\phi_{\Gamma}(r)$ through the stationary Schrödinger equation [18]:

$$
V_{\Gamma}(r)-E_{\Gamma}=\frac{1}{m_{q}} \frac{\nabla^{2} \phi_{\Gamma}(r)}{\phi_{\Gamma}(r)}
$$

where $m_{q}$ is the quark kinetic mass and $\nabla^{2}$ is defined by the discrete Laplacian with nearest-neighbor points. The energy eigenvalue $E_{\Gamma}$ of the stationary Schrödinger equation is supposed to be $M_{\Gamma}-2 m_{q}$. Here we note that this definition of the potential itself requires information of the quark mass $m_{q}$, while the rest mass of the heavy quarkonium state $M_{\Gamma}$ can be determined by the standard hadron spectroscopy.

The central potential calculated from $1 S$ quarkonium states can be decomposed into the spin-independent and spin-dependent parts: $V_{\Gamma}(r)=V_{q \bar{q}}(r)+V_{\text {spin }}(r) \mathbf{S}_{q} \cdot \mathbf{S}_{\bar{q}}$, where $V_{q \bar{q}}$ represents the spin-independent central potential while $V_{\text {spin }}$ corresponds to the spin-spin potential. For the PS and V channels, the spin operator $\mathbf{S}_{q} \cdot \mathbf{S}_{\bar{q}}$ can be easily replaced by expectation values $-3 / 4$ and $1 / 4$, respectively. Therefore, the potential $V_{q \bar{q}}$ can be evaluated by a linear combination of potentials calculated from the PS and V channels as $V_{q \bar{q}}(r)=\frac{1}{4}\left[V_{\mathrm{PS}}(r)+3 V_{\mathrm{V}}(r)\right]$.

As we previously pointed out, the quark kinetic mass $m_{q}$ is a key ingredient in order to calculate the $q \bar{q}$ potential defined in Eq. (4) from the BS wave function. How can we determine the quark mass? In the initial attempt [8], $m_{q}$ was approximately evaluated by half of the vector quarkonium mass $M_{V} / 2$. However, such an approximate treatment loses a proper quark-mass dependence of the $q \bar{q}$ potential, which guarantees that the potential defined here is smoothly connected to the static $q \bar{q}$ potential from Wilson loops in the $m_{q} \rightarrow \infty$ limit.

We may alternatively determine the quark mass from the gauge dependent pole mass, which can be measured by the quark two-point function in the Landau gauge. We instead propose a novel method which is applicable even in the Coulomb gauge as follows. We first consider the spin-dependent potential, which is given by

$$
V_{\mathrm{spin}}(r)-\Delta E_{\mathrm{hyp}}=\frac{1}{m_{q}}\left(\frac{\nabla^{2} \phi_{\mathrm{V}}(r)}{\phi_{\mathrm{V}}(r)}-\frac{\nabla^{2} \phi_{\mathrm{PS}}(r)}{\phi_{\mathrm{PS}}(r)}\right),
$$

where $\Delta E_{\text {hyp }}$ denotes a difference between energy eigenvalues of the PS and $\mathrm{V}$ channels. Indeed, the value of $\Delta E_{\text {hyp }}$ is nothing but hyperfine mass splitting $M_{\mathrm{V}}-M_{\mathrm{PS}}$.

According to the $1 / m_{q}$ expansion approach, we may expect to have the condition of $\lim _{r \rightarrow \infty} V_{\text {spin }}(r)=0$,
TABLE I: Results of the quark mass $m_{q}$, the Cornell parameters $A, \sigma$, and the ratio $A / \sigma$ in this approach. Their extrapolated values to the $m_{q} \rightarrow \infty$ limit are also compared with the Wilson loop results taken from Ref. [7.

\begin{tabular}{|ccccc|}
\hline \hline$\kappa$ & $a m_{q}$ & $A$ & $a^{2} \sigma$ & $A / a^{2} \sigma$ \\
\hline 0.11456 & $0.493(18)$ & $0.663(23)$ & $0.0477(28)$ & $13.9(7)$ \\
0.10190 & $0.833(31)$ & $0.470(16)$ & $0.0435(25)$ & $10.8(6)$ \\
0.09495 & $1.006(41)$ & $0.430(16)$ & $0.0426(27)$ & $10.1(6)$ \\
0.08333 & $1.288(30)$ & $0.381(10)$ & $0.0435(18)$ & $8.8(4)$ \\
0.07490 & $1.484(22)$ & $0.360(7)$ & $0.0443(13)$ & $8.1(3)$ \\
0.06667 & $1.720(18)$ & $0.341(6)$ & $0.0442(11)$ & $7.7(3)$ \\
\hline- & $\infty$ & $0.236(39)$ & $0.0465(34)$ & $6.1(1.1)$ \\
\hline Wilson loop & $0.281(5)$ & $0.0466(2)$ & $6.03(11)$ \\
\hline \hline
\end{tabular}

which implies that there is no long-range correlation and no irrelevant constant term in the spin-dependent potential [5, 7. We thus can estimate the quark kinetic mass $m_{q}$ through the following formula:

$$
m_{q}=\lim _{r \rightarrow \infty} \frac{1}{\Delta E_{\mathrm{hyp}}}\left(\frac{\nabla^{2} \phi_{\mathrm{PS}}(r)}{\phi_{\mathrm{PS}}(r)}-\frac{\nabla^{2} \phi_{\mathrm{V}}(r)}{\phi_{\mathrm{V}}(r)}\right),
$$

where $\Delta E_{\mathrm{hyp}}=M_{\mathrm{V}}-M_{\mathrm{PS}}$ is measured by the standard hadron spectroscopy. As a result, one can selfconsistently determine both the spin-independent and spin-dependent $q \bar{q}$ potentials, and also the quark kinetic mass within a single set of four-point correlation functions.

To verify our new proposal, we have performed quenched lattice QCD simulations on a lattice $L^{3} \times T=$ $32^{3} \times 48$ with the standard single-plaquette gauge action at $\beta=6 / g^{2}=6.0$, which corresponds to a lattice cutoff of $a^{-1} \approx 2.1 \mathrm{GeV}(a \approx 0.093 \mathrm{fm})$. The spatial lattice size corresponds to $L a \approx 3 \mathrm{fm}$. We fix the lattice to Coulomb gauge. The heavy-quark propagators are computed using the relativistic heavy-quark (RHQ) action with relevant one-loop coefficients of the RHQ [14, 15. The RHQ action utilized here is a variant of the Fermilab approach [16] and can remove large discretization errors introduced by large quark mass.

To examine the infinitely heavy-quark limit, we adopt the six values of the hopping parameter $\kappa$, which cover the range of the spin-averaged mass of $1 S$ quarkonium states $M_{\text {ave }}=\frac{1}{4}\left(M_{\mathrm{PS}}+3 M_{\mathrm{V}}\right)=1.97-5.86 \mathrm{GeV}$. We calculate quark propagators with a wall source which is located at $t_{\mathrm{src}} / a=4$. Dirichlet boundary conditions are imposed for time direction. Our results are analyzed on 150 configurations for every hopping parameters. In this letter, we use only the on-axis data of the BS wave function since the off-axis data may suffer more from the rotational symmetry breaking effect.

First, in Fig. 1. we plot a difference of ratios of $\nabla^{2} \phi_{\mathrm{V}} / \phi_{\mathrm{V}}$ and $\nabla^{2} \phi_{\mathrm{PS}} / \phi_{\mathrm{PS}}$ as a function of spatial distance $r$ at $\kappa=0.10190$, which is close to the charm quark mass [17, as a typical example. The ratios of $\nabla^{2} \phi_{\Gamma} / \phi_{\Gamma}$ are evaluated by a weighted average of data points in the 


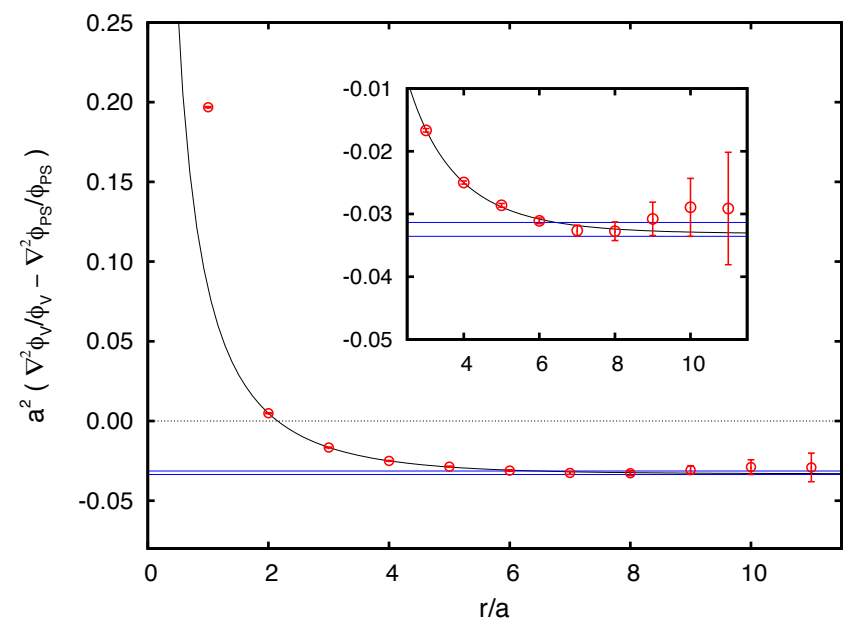

FIG. 1: A typical result of $\nabla^{2} \phi_{\mathrm{V}} / \phi_{\mathrm{V}}-\nabla^{2} \phi_{\mathrm{PS}} / \phi_{\mathrm{PS}}$ as a function of spacial distance $r$, which is calculated at a quark mass close to the charm quark mass. A nonzero constant shift observed at large distances corresponds to the value of $-m_{q} \Delta E_{\text {hyp }}$ (solid line), which is determined by a weighted average of data points at $r / a=7-11$ in this case. A solid curve is a fit to the Yukawa plus constant form.

range of $\left(t-t_{\mathrm{src}}\right) / a=21-23$. At a glance, the value of $\nabla^{2} \phi_{\mathrm{V}} / \phi_{\mathrm{V}}-\nabla^{2} \phi_{\mathrm{PS}} / \phi_{\mathrm{PS}}$ certainly reaches a nonzero constant value at large distances, which turns out to be the value of $-m_{q} \Delta E_{\mathrm{hyp}}$. The values of $m_{q} \Delta E_{\mathrm{hyp}}$ are evaluated by a weighted average of data points in the range where $V_{\text {spin }}(r)$ should vanish. We then obtain the quark kinetic masses from the long-distance asymptotic values of $\nabla^{2} \phi_{\mathrm{V}} / \phi_{\mathrm{V}}-\nabla^{2} \phi_{\mathrm{PS}} / \phi_{\mathrm{PS}}$ divided by the measured hyperfine splitting $\Delta E_{\text {hyp }}$.

Apart from the vertical scale and offset, Fig. 1 1 exhibits the $r$ dependence of the spin-spin potential $V_{\text {spin }}(r)$. The potential is quickly dumped at large distances as mentioned previously. Although the spin-spin interaction based on one-gluon exchange like the Fermi-Breit interaction of QED is described by a short-range $\delta$-function potential, it is evident that the obtained spin-spin potential is a repulsive potential with some finite range. Indeed, the long-range screening observed here is simply accommodated by exponential-type fitting functions $\exp \left(-r^{m}\right) / r^{n}$. If we adopt the Yukawa form $(m=n=1)$ with a constant shift $c e^{-\alpha r} / r+d$ to fit our data of $V_{\text {spin }}(r)$, we can get a reasonable fit over the range of $r / a$ from 2 to 11 . The fitting result is displayed as a solid curve in Fig 1. In addition, the parameter $\alpha$ is very sensitive to the quark mass. It increases with the quark mass in the measured mass region of $m_{q}=1.0-3.6 \mathrm{GeV}$. We plan to present more detail on the spin-spin potential in a separate publication.

Using the quark kinetic mass determined here, we can properly calculate the spin-independent interquark potential $V_{q \bar{q}}(r)$ from the BS wave functions without any

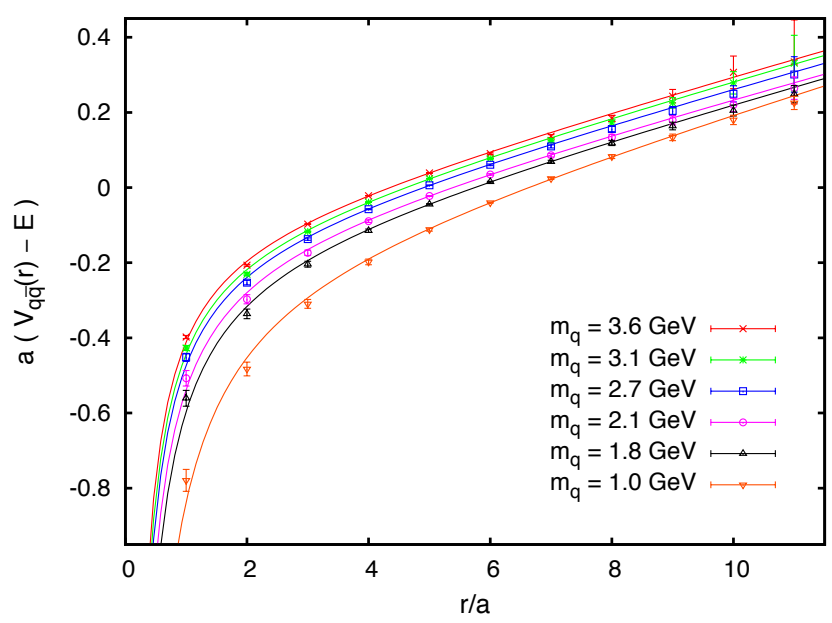

FIG. 2: The interquark potential calculated from the $q \bar{q}$ BS amplitude at finite quark masses covering the range from 1.0 to $3.6 \mathrm{GeV}$. For clarity of the figure, the constant energy shift $E$ is not subtracted. Each curve represents the fit result with the Cornell parametrization defined in Eq. (7).

ambiguity. Figure 2 displays results of our potential $V_{q \bar{q}}$ obtained at several quark masses. For clarity of the figure, the constant energy shift $E$, which corresponds to the value of $M_{\mathrm{av}}-2 m_{q}<0$, is not subtracted in Fig. 2 . The resulting $q \bar{q}$ potentials at finite quark masses exhibit the linearly rising potential at large distances and the Coulomb-like potential at short distances as originally reported in Ref 8 .

We simply adopt the Cornell parametrization for fitting our data:

$$
V_{q \bar{q}}(r)=-\frac{A}{r}+\sigma r+V_{0}
$$

with the Coulombic coefficient $A$, the string tension $\sigma$, and a constant $V_{0}$. It is found that the Cornell potential describes well the $q \bar{q}$ potential even at a lighter quark mass than the charm quark mass. Although such a light mass region is beyond the radius of convergence for the systematic $1 / m_{q}$ expansion, the finite $m_{q}$ corrections could be nonperturbatively encoded into the Cornell parameters in this approach. All fits are performed over the range $1 \leq r / a \leq 11$. Fit results for $A$ and $\sigma$ are summarized in Table I.

In Fig. 3, we show the quark-mass dependence of $A / \sigma$, $A$ and $\sigma$ as functions of $1 / m_{q}$. First, regardless of the definition of $m_{q}$, the ratio of $A / \sigma$ in the top figure indicates that the $q \bar{q}$ potential calculated from the BS wave function smoothly approaches the potential obtained from Wilson loops in the infinitely heavy-quark limit. Indeed, the $m_{q} \rightarrow \infty$ extrapolation by a quadratic fit (solid curve) with respect to $1 / m_{q}$ to four heaviest points (filled squares), where $1 / m_{q}$ in lattice units is less than unity, is consistent with the value obtained from Wilson loops. 


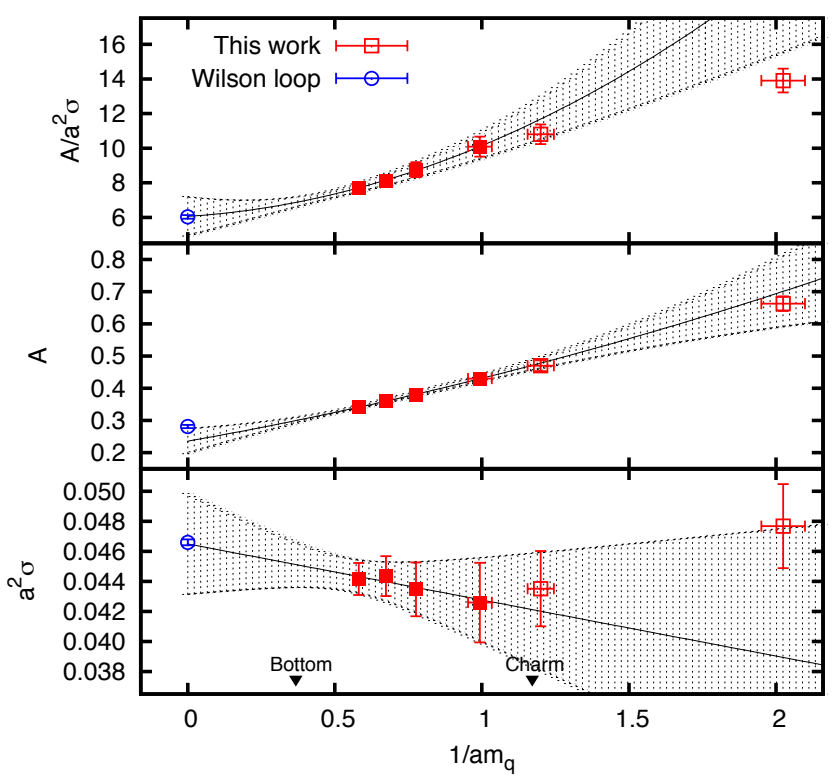

FIG. 3: The quark-mass dependence of $A / \sigma, A$, and $\sigma$ as functions of $1 / m_{q}$. We perform the extrapolation towards the $m_{q} \rightarrow \infty$ limit (solid curves) of $A / \sigma, A$, and $\sigma$ with a simple polynomial function in $1 / m_{q}$. For $\sigma$, a linear fit with respect to $1 / m_{q}$ is enough to describe the data with reasonable $\chi^{2} /$ d.o.f, while quadratic fits are used for $A / \sigma$ and $A$. The results given by Wilson loops are also included as open circles.

If we pay attention to the quark-mass dependence of each of the Cornell parameters separately, we observe that, although the Coulombic parameter $A$ depends on the quark mass significantly, there is no appreciable dependence of the quark mass on the string tension $\sigma$. These observations agree with several features of the $1 / m_{q}$ corrections to the static $q \bar{q}$ potential found in Refs. [6, 7]. The long-range part of the potential characterized by the string tension receives no correction up to $\mathcal{O}\left(1 / m_{q}^{2}\right)$ in the Wilson loop formalism, while the $1 / m_{q}$ correction on the Coulomb term starts at $\mathcal{O}\left(1 / m_{q}\right)$. Here we recall that our way of determining the interquark potential with the proper quark mass defined in Eq. (6) is responsible for desirable quark-mass dependence observed here.

We finally evaluate the values of $A$ and $\sigma$ in the infinitely heavy-quark limit by quadratic and linear fits with respect to $1 / m_{q}$, respectively. Extrapolated values at $m_{q} \rightarrow \infty$ are again consistent with those of the Wilson loop result. The extrapolation curve and line are also displayed as a solid curve and line in Fig. 3

In this letter, we have proposed the new method to determine the interquark potential $V_{q \bar{q}}(r)$ at finite quark mass for heavy quarkonia from lattice QCD. The selfconsistent determination of the quark kinetic mass $m_{q}$ plays an essential role on how to define the interquark potential from the $q \bar{q} \mathrm{BS}$ amplitude. Using quenched lattice QCD, we have demonstrated that the spin-independent central potential defined in this method smoothly approaches the static $q \bar{q}$ potential given by Wilson loops in the infinitely heavy-quark limit. We also found that our defined potential, which is well described by the Cornell parametrization even at finite quark mass, exhibits the desired quark-mass dependence of the Cornell parameters, which agrees well with what is expected from results obtained in the systematic $1 / m_{q}$ expansion approach. This suggests that our interquark potential properly accounts for all orders of $1 / m_{q}$ corrections and its nature is validated at least up to the charm quark sector. We also stress that this new method allows us to calculate the spin-dependent potential with high accuracy. We will next apply to dynamical calculation of the interquark potential at the charm quark mass, of which precise knowledge is important especially for physics of higher charmonia 4]. Such planning is now underway.

We would like to thank T. Hatsuda for helpful suggestions, H. Iida and Y. Ikeda for fruitful discussions. T.K. is supported by Grant-in-Aid for the Japan Society for Promotion of Science (JSPS) (No. 22-7653). S.S. is supported by the JSPS Grant-in-Aids for Scientific Research (C) (No. 19540265) and Scientific Research on Innovative Areas (No. 21105504).

* Electronic address: kawanai@nt.phys.s.u-tokyo.ac.jp

$\dagger$ Electronic address: ssasaki@phys.s.u-tokyo.ac.jp

[1] For a review, see G. S. Bali, Phys. Rept. 343, 1 (2001).

[2] E. Eichten et.al., Phys. Rev. Lett. 36, 500 (1976).

[3] S. Godfrey and N. Isgur, Phys. Rev. D 32, 189 (1985).

[4] T. Barnes, S. Godfrey and E. S. Swanson, Phys. Rev. D 72, 054026 (2005).

[5] N. Brambilla et al., Rev. Mod. Phys. 77, 1423 (2005).

[6] Y. Koma, M. Koma and H. Wittig, Phys. Rev. Lett. 97, 122003 (2006).

[7] Y. Koma and M. Koma, Nucl. Phys. B 769, 79 (2007).

[8] Y. Ikeda and H. Iida, arXiv:1011.2866 and 1102.2097.

[9] N. Ishii, S. Aoki and T. Hatsuda, Phys. Rev. Lett. 99, 022001 (2007).

[10] S. Aoki, T. Hatsuda and N. Ishii, Prog. Theor. Phys. 123 (2010) 89

[11] B. Velikson and D. Weingarten, Nucl. Phys. B 249, 433 (1985).

[12] R. Gupta et al., Phys. Rev. D 48, 3330 (1993).

[13] M. Lüscher, Nucl. Phys. B 354, 531 (1991).

[14] S. Aoki, Y. Kuramashi and S. I. Tominaga, Prog. Theor. Phys. 109, 383 (2003).

[15] Y. Kayaba et al. [CP-PACS Collaboration], JHEP 0702, 019 (2007).

[16] A. X. El-Khadra et al., Phys. Rev. D 55, 3933 (1997).

[17] T. Kawanai and S. Sasaki, Phys. Rev. D 82, 091501 (2010).

[18] Although the potential defined in this way is generally non-local, it becomes a local potential at low energies in a sense of the derivative expansion. A detailed discussion can be found in Ref. [10. 